\title{
NEUROLOGICAL PATIENT SAFETY: CLINICAL PRACTICE GUIDE
}

\section{ABSTRACT}

INTRODUCTION: The promotion of patient safety culture is essential for the qualification of the work process and the care provided. However, teams need to be engage and committed so that this care is patient-centered and based on evidence-based practice. Clinical practice guide is a tool that helps and contributes to safe care, so the aim of this study was the creation of a nursing clinical practice guide directed to the care of neurological patients submitted to external ventricular bypass. METHOD: A research on technological development in health for the development of the nursing clinical practice guide. The Guide for the Construction of Nursing Care Protocols that includes the essential items for a quality material guided this research. The evidence that supported the construction of this product was originated from a scoping review previously performed. Each evidence was assess for its quality level according Grade System. RESULTS: The nursing clinical practice guideline presents an introduction to the theme and the patient profile that is direct, including algorithms that help and speed up the team's decision-making. In addition, it presents actions based on the studies selected in the systematic review. Twenty nursing care were considered essential for a standardized, qualified and safe practice, optimizing the work process of the care team. CONCLUSION: The promotion of patient safety is a fundamental component for the qualification of care and responsibility of all professionals. Clinical practice guide are consider health technologies serving as tools that underlie and qualify the process of safe, effective and quality care. The protocols help teams to develop and promote the culture of patient safety in their work dynamics, in order to insert it as a natural and intrinsic process in daily praxis.

Key words: external ventricular bypass; neurological patients; Clinical Practice Guide Nursing Care; .Patient safety. 